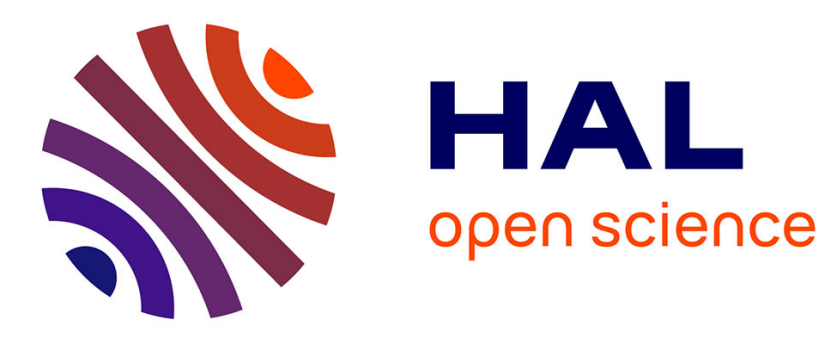

\title{
Time Reversal of Water Waves
}

\author{
A. Przadka, S. Feat, P. Petitjeans, V. Pagneux, A. Maurel, M. Fink
}

\section{To cite this version:}

A. Przadka, S. Feat, P. Petitjeans, V. Pagneux, A. Maurel, et al.. Time Reversal of Water Waves. Physical Review Letters, 2012, 109 (6), 10.1103/PhysRevLett.109.064501 . hal-02454168

\section{HAL Id: hal-02454168 https://hal.science/hal-02454168}

Submitted on 24 Jan 2020

HAL is a multi-disciplinary open access archive for the deposit and dissemination of scientific research documents, whether they are published or not. The documents may come from teaching and research institutions in France or abroad, or from public or private research centers.
L'archive ouverte pluridisciplinaire HAL, est destinée au dépôt et à la diffusion de documents scientifiques de niveau recherche, publiés ou non, émanant des établissements d'enseignement et de recherche français ou étrangers, des laboratoires publics ou privés. 


\title{
Time Reversal of Water Waves
}

\author{
A. Przadka, S. Feat, and P. Petitjeans \\ Physique et Mécanique des Milieux Hétérogènes, PMMH, UMR CNRS 7636-ESPCI-UPMC Université Paris \\ 6-UPD Université Paris 7, 75005 Paris, France
}

\author{
V. Pagneux \\ Laboratoire d'Acoustique de l'Université du Maine, UMR CNRS 6613, Avenue Olivier Messiaen, 72085 Le Mans, France
}

\author{
A. Maurel and M. Fink \\ Institut Langevin, ESPCI \& CNRS, UMR CNRS 7587, 10 rue Vauquelin, 75005 Paris, France
}

(Received 2 April 2012; published 9 August 2012)

\begin{abstract}
We present time reversal experiments demonstrating refocusing of gravity-capillary waves in a water tank cavity. Owing to the reverberating effect of the cavity, only a few channels are sufficient to reconstruct the surface wave at the point source, even if the absorption is not negligible. Space-timeresolved measurements of the waves during the refocusing allow us to quantitatively demonstrate that the quality of the refocusing increases linearly with the number of reemitting channels. Numerical simulations corresponding to water waves at larger scales, with negligible damping, indicate the possibility of very high quality refocusing.
\end{abstract}

DOI: 10.1103/PhysRevLett.109.064501

PACS numbers: 47.35.- $\mathrm{i}$

Time reversal of acoustic, elastic, and electromagnetic waves has been extensively studied in recent years [1,2]. In a standard time reversal (TR) experiment, waves generated by a source are first measured by an array of antennas positioned around the source and then time reversed and simultaneously rebroadcasted by the same antenna array. Due to the time invariance of the wave process, the reemitted energy will focus back on the original source, whatever the complexity of the propagation medium [3]. According to diffraction theory, an exact control of the wave would require the measurement and time reversal of both the wave and its normal derivative along a closed surface. This would require a large number of antennas and time reversal channels. However, it has been shown that the reversal remains quite good even when these conditions are not fulfilled by the experiment [4]. One surprising result that was demonstrated both for elastic [5-7] and electromagnetic waves [2] was that, when a broadband wave field is trapped inside a reverberating cavity, a one-channel time reversal antenna is enough to refocus back onto the source. In this case, the refocusing quality depends directly on the number of cavity modes that are excited by the source. To better resolve these modes, breaking the symmetries of the cavity avoids mode degeneracy which improves the refocusing [6].

The present work concentrates on the application of time reversal to the focusing of water waves in a laboratory tank which plays the role of the reverberating cavity. Although water waves are ubiquitous and easily observable, they have never been tested in a time reversal experiment (for theoretical formulation of time reversal for water waves, see Ref. [8]). This is certainly due to their complexity: to cite Richard Feynman, "[water waves] that are easily seen by everyone and which are usually used as an example of waves in elementary courses [...] are the worst possible example, because they are in no respects like sound and light; they have all the complications that waves can have" [9]. Water waves are scalar waves; that refers to the evolution of small perturbation of the height of fluid $\eta(\mathbf{r}, t)$ under the action of gravity and surface tension. They are dispersive by nature, nonlinear when generated with standard wavemakers, and they experience strong damping at the scale of laboratory experiments. The effect of dispersion on the time reversal process has already been studied in time reversal experiments for guided elastic waves $[5,10]$; these waves are dispersionless in free space, and the dispersion is due only to the reflection on the boundaries of the waveguide. In the case of water waves, the dispersion is intrinsic but preserves the time reversal invariance (obviously, not taking the damping into account).

The effect of the nonlinearities has been experimentally studied in Ref. [11] for acoustic waves where it has been shown that the time reversal invariance is preserved as long as nonlinearities do not create dissipation, i.e., as long as the propagation distance is smaller than the shock distance. In the case of water waves, the effect of nonlinearities has been theoretically studied displaying that refocusing is robust with respect to nonlinearities, when small but not negligible [8]. Eventually, the main complication in a water wave TR experiment is the damping. The latter has various origins: (i) the viscosity of the fluid that produces a bulk damping, (ii) the bottom friction (water waves produce oscillations at the bottom), which occurs when the wavelength is not small compared to the fluid depth since 
the penetration depth of the wave is given by the wavelength, (iii) the film surface effect that is able to create an important friction just beneath the surface of the liquid (the calming effect of oil on troubled waters [12]), and (iv) the walls of the water tank. The damping may break time reversal invariance if it is too strong. It was already shown by our group in Ref. [13], where the same experimental setup was used, that no surface film effect is present and that the damping coefficient is comparable to the one of pure water.

In this Letter, we show for the first time an experimental proof of time reversal of gravity-capillary waves. The experiment is conducted in a water tank cavity to take advantage of multiple reflections on the boundaries. The influence of the number of channels in the time reversal mirror is studied and it allows us to show that a small number of channels is sufficient to obtain the TR refocusing, owing to the reverberating effect of the cavity.

The reverberating tank is filled with water with depth at rest $H=10 \mathrm{~cm}$. The dimension of the rectangular tank is $53 \times 38 \mathrm{~cm}^{2}$ with obstacles placed in order to break the spatial symmetries (see Fig. 1). The waves are generated by using a vertical conical vibrator and recorded by using an optical method (note that this differs from TR experiments in acoustics that use the same transducer to record and to regenerate the wave). We perform a typical one-channel TR experiment in two steps. In the first step of emission, a wave packet is generated from a conical vibrator that can be considered as a point source. In a second step, the signal recorded at a receiver point is time reversed and reemitted.

The time reversed wave is expected to refocus spatially at the source point and refocus temporally at the recompression time. As previously stressed, the key point to ensure TR refocusing is the number of cavity modes that

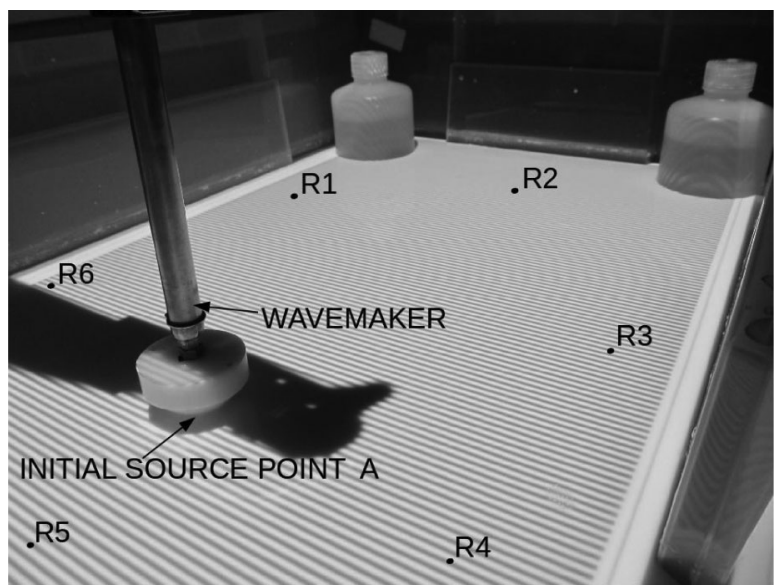

FIG. 1. Water tank used in the experiment. Point $A$ is at the initial source position. Note the fringes used for the Fourier transform profilometry technique. Points $R_{1}$ to $R_{6}$ correspond to the positions of the different channels of the time reversal mirror. At each of these channel positions, the wave is measured and can be reemitted after the time reversal operation. the wave packet has been able to excite in the first step of the TR.

After a few experimental trials, the authors found the central frequency $f_{0}=5 \mathrm{~Hz}$ the best to obtain good refocusing in time and space. This is a compromise between the bulk dissipation that grows with a frequency increase and the bottom friction that becomes significant while decreasing frequency [note: no significant peaks in the low frequency region in Fig. 2(b)]. Figure 2(a) shows the signal recorded at one point (point $R_{1}$ in Fig. 1) when a one-period sinusoidal pulse centered at $f_{0} \mathrm{~Hz}$ is generated at the initial source position. The duration of the signal is typically $20 \mathrm{~s}$, corresponding both to reverberating effects and linear dispersion effects. The latter is given by the linear dispersion relation for water wave propagation (taking into account the effects of finite depth $H$ and surface tension $\gamma$ ):

$$
\omega^{2}=\left(g k+\frac{\gamma}{\rho} k^{3}\right) \tanh k H,
$$

where $k$ denotes the complex wave number, $g$ the gravity acceleration, and $\rho$ the water density. The wavelength at central frequency $f_{0}$ is $\lambda=6 \mathrm{~cm}$. The magnitude of nonlinearity of the waves based on the maximum measured gradient of surface elevation was found to be $\epsilon=0.13$. The attenuation is such that the wave can propagate over roughly 100 wavelengths, i.e., about 10 to 20 times the length scale of the cavity. This is consistent with the $20 \mathrm{~s}$ duration of the time signal recorded at one point in the cavity [Fig. 2(a)] since the phase velocity at the central frequency is $0.33 \mathrm{~m} / \mathrm{s}$ corresponding to $12-17$ reflections from the boundaries. The spectrum of the signal recorded
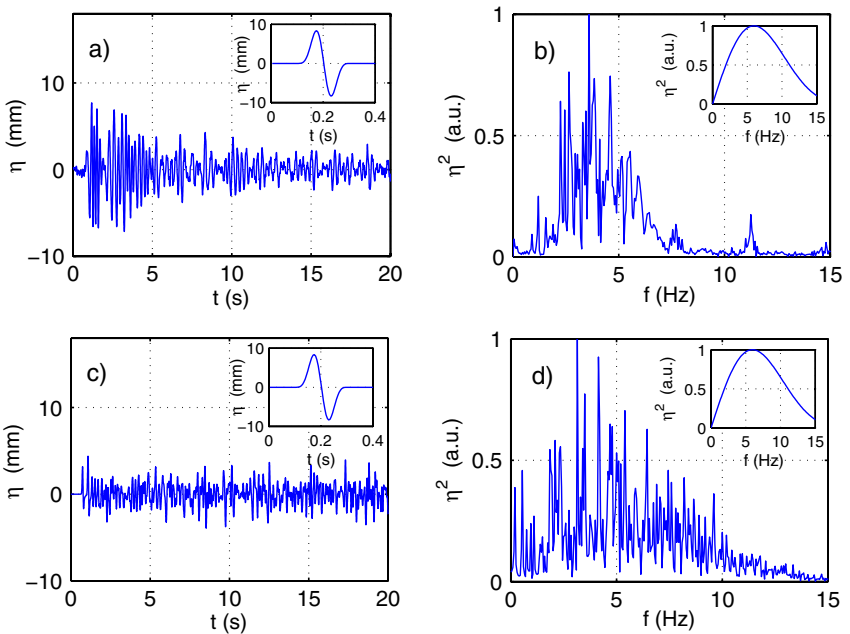

FIG. 2 (color online). (a) Experimental measurement of the temporal evolution of the surface elevation $\left[\eta\left(\mathbf{r}_{1}, t\right)\right]$ during the forward propagation after emission from point $A$. The inset shows the signal emitted from point $A$. (b) Corresponding spectrum; the inset shows the spectrum of the signal emitted from point $A$. (c),(d) Same representation from numerical simulations of the wave equation. 
during the direct propagation is shown in Fig. 2(b). It presents several peaks (one can count roughly 20 peaks) corresponding to the eigenmodes of the cavity that have been excited. Although the initial pulse covers a broadband frequency range $[0,15] \mathrm{Hz}$, the signal recorded is limited to frequencies smaller than about $10 \mathrm{~Hz}$. We have checked that this is an effect of the attenuation: direct numerical simulations of the 2D wave equation in the same geometry but omitting the attenuation give a spectrum with around 100 cavity modes excited in the whole range $[0,15] \mathrm{Hz}$ [Figs. 2(c) and 2(d)].

We now investigate the refocusing. The perturbation of the surface elevation $\eta(\mathbf{r}, t)$ is measured in time and in space during the wave propagation using an optical method (Fourier transform profilometry) that has been adapted recently to water wave measurements [13-16]. Fourier transform profilometry is used to measure the whole pattern of surface elevation $\eta(\mathbf{r}, t)$ at each time of the reverse propagation. This has been done in one-channel experiments $(N=1)$. Although the spatial refocusing and temporal recompression are visible (Fig. 3), it is not possible to distinguish the converging wave fronts before the recompression and the diverging wave fronts after recompression in these one-channel experiments.

To improve the refocusing, it is possible to increase the number of channels. In a time reversal experiment with multiple channels, the signal emitted from the source point is recorded at $N$ receiver points. The TR signal is then reemitted, simultaneously, from the $N$ receiver points. If the $N$ receiver points are uncorrelated, it is meant to improve the refocusing since the wave experiences many different trajectories in the cavity. In our experiment, rather than using $N$ wave generators to reemit the signal, the $N$-channel TR has been done with just one wave generator. This is possible by exploiting the linearity of the problem which implies that the wave field excited by $N$ generators is equal to the sum of the $N$ wave fields excited by each generator alone. We have checked this linearity by comparing the time signal recorded at the refocalization point when using two channels and when summing the two signals obtained in two single-channel experiments. The temporal signals in both cases coincide, with less than $10 \%$ discrepancy in a $10 / f_{0}$ time window centered at the recompression time. Six different positions of the receiver point have been used (points $R_{1}$ to $R_{6}$ in Fig. 1). Figure 4 shows a time sequence of the reverse propagation for the six-channel TR. As expected, the peak at the recompression time is much higher than in the one-channel TR, confirming that the channels are uncorrelated. Besides, the converging and diverging wave fronts, before and after the recompression time, are visible.

To gain insights in the study of the quality of the refocusing, we want to inspect both the effect of the number of channels and the effect of the damping. To characterize the quality of the spatial refocusing, we define

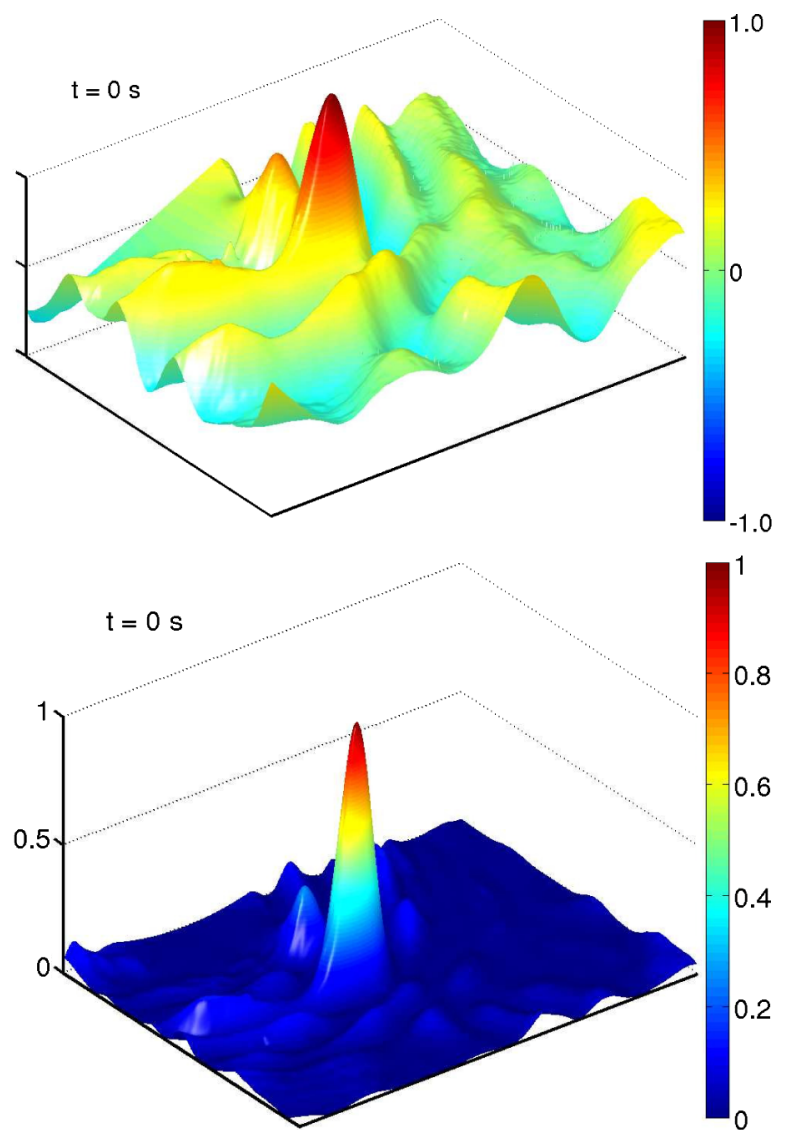

FIG. 3 (color online). Measured amplitude $[\eta(\mathbf{r}, t=0)]$ and intensity $\left[\eta(\mathbf{r}, t=0)^{2}\right]$ of the time reversed wave around the initial source position (point $A$ ) where the wave is refocused at the recompression time $(t=0)$ in a one-channel TR experiment $(N=1)$. The spatial range around the refocusing point $A$ is $34 \times 26 \mathrm{~cm}^{2}$.

a peak-to-noise ratio (PNR) at the recompression time as the ratio of the maximum intensity at the focal spot to the mean sidelobe intensity. Experimental results are shown in Fig. 5 (black circles). With a single channel, the peak-tonoise ratio is typically 20 , a value that is related to the number of cavity modes that can be resolved from the spectrum at the receiver point in Fig. 2. With several channels, the PNR increases linearly with the number $N$ of channels [6]. Although this behavior is expected without damping, it was not obvious and was verified with the typical range of damping of our experiment. The insets of Fig. 5 show the temporal recompression for $N=1$ and $N=6$ at the refocusing point $A$. The refocusing is clearly visible in the one-channel time reversal experiment but with higher temporal sidelobes than with six channels. Note that these temporal signals allow us also to define a peak-to-noise ratio and we observed that PNRs either defined in space or in time have roughly the same values. Varying the damping is more difficult. To perform experiments where the damping effect is negligible would necessitate a much larger size of the cavity (e.g., the size of 

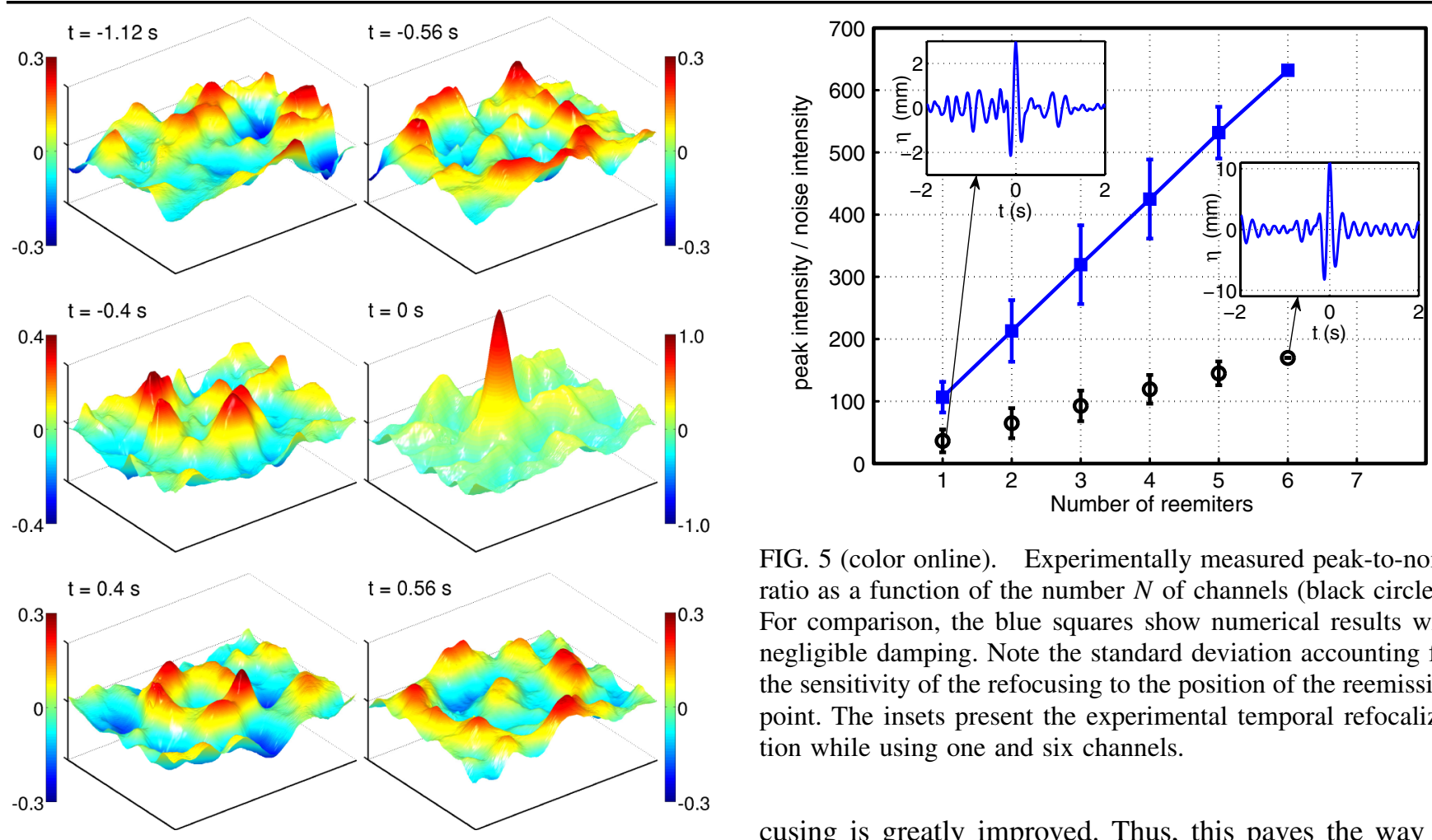

FIG. 5 (color online). Experimentally measured peak-to-noise ratio as a function of the number $N$ of channels (black circles). For comparison, the blue squares show numerical results with negligible damping. Note the standard deviation accounting for the sensitivity of the refocusing to the position of the reemission point. The insets present the experimental temporal refocalization while using one and six channels.

cusing is greatly improved. Thus, this paves the way to applications in the context of water waves in the sea, with very small damping, where a very high quality of refocusing is expected.

The authors thank the Agence Nationale de la Recherche for its funding under Grant No. Tourbillonde ANR-08BLAN-0108-02.

respectively, for negative and positive time. The spatial range around the refocusing point $A$ is $34 \times 26 \mathrm{~cm}^{2}$.

a swimming pool) because the attenuation per wavelength decreases with the frequency [17]. Therefore, numerical simulations have been used to model the case with negligible damping. The results are shown in Fig. 5 (blue squares) where computations have been done by taking the same protocol as in the experiment. The same trends, as in the experiment, are observed: (i) for $N=1$, the PNR is equal to 100 and is given by the number of excited cavity modes that can be resolved in the spectrum in Fig. 2 and (ii) the PNR increases linearly with $N$. With about 20 excited modes in our laboratory experiments, versus the 100 modes obtained in the numerics, it appears that the refocusing can be significantly reduced because of the attenuation occurring at that laboratory scale.

Our experiments illustrate the feasibility of a fewchannel time reversal refocusing for gravity-capillary waves. This has been performed in a well controlled laboratory context that allows quantitative measurements, simultaneously, in time and space. At this laboratory scale, with centimetric wavelengths, the quality of the refocusing is limited by the damping due to viscous effects, but it is not suppressed. At larger scales, viscous damping highly decreases and numerical simulations show that the refo-

[1] M. Fink, Phys. Today 50, No. 3, 34 (1997).

[2] G. Lerosey, J. de Rosny, A. Tourin, A. Derode, G. Montaldo, and M. Fink, Phys. Rev. Lett. 92, 193904 (2004).

[3] M. Fink, Geophysics 71, SI151 (2006).

[4] M. Fink, D. Cassereau, A. Derode, C. Prada, P. Roux, M. Tanter, J.-L. Thomas, and F. Wu, Rep. Prog. Phys. 63, 1933 (2000).

[5] C. Draeger and M. Fink, Phys. Rev. Lett. 79, 407 (1997).

[6] C. Draeger and M. Fink, J. Acoust. Soc. Am. 105, 611 (1999).

[7] C. Draeger, J.-C. Aime, and M. Fink, J. Acoust. Soc. Am. 105, 618 (1999).

[8] J. P. Fouque and A. Nachbin, Multiscale Model. Simul. 1, 609 (2003).

[9] R. P. Feynman, R. B. Leighton, and M. Sands, The Feynman Lectures on Physics (Addison-Wesley, Reading, MA, 1963), Vol. I, Chaps. 51-54.

[10] R. K. Ing and M. Fink, IEEE Trans. Ultrason. Ferroelectr. Freq. Control 45, 1032 (1998).

[11] M. Tanter, J.-L. Thomas, F. Coulouvrat, and M. Fink, Phys. Rev. E 64, 016602 (2001).

[12] P. S. Behroozi, K. Cordray, W. Griffin, and F. Behroozi, Am. J. Phys. 75, 407 (2007). 
[13] Recent improvements of the technique for the signal processing and the choice of the painting particles can be found, respectively, in G. Lagubeau et al. (to be published) and in A. Przadka, B. Cabane, V. Pagneux, A. Maurel, and P. Petitjeans, Exp. Fluids 52, 519 (2011).

[14] A. Maurel, P. Cobelli, V. Pagneux, and P. Petitjeans, Appl. Opt. 48, 380 (2009); P. Cobelli, A. Maurel, V. Pagneux, and P. Petitjeans, Exp. Fluids 46, 1037 (2009).
[15] P. Cobelli, P. Petitjeans, A. Maurel, V. Pagneux, and N. Mordant, Phys. Rev. Lett. 103, 204301 (2009).

[16] P. Cobelli, V. Pagneux, A. Maurel, and P. Petitjeans, Europhys. Lett. 88, 20006 (2009); J. Fluid Mech. 666, 445 (2011).

[17] M. J. Lighthill, Waves in Fluids (Cambridge University Press, Cambridge, England, 2001). 